\title{
Correlation and response relevance in sequence learning
}

\author{
Josephine Cock $\cdot$ Beat Meier
}

Received: 1 February 2012/ Accepted: 12 June 2012/Published online: 6 July 2012

(C) Springer-Verlag 2012

\begin{abstract}
We tested the effects of introducing a secondary sequence into the serial reaction time task. Specifically, we examined the role of correlated streams of information and response relevance. In the first experiment, the order of stimulus locations was correlated with the order of key press responses in the conventional way. A symbol-identity sequence, of a different length, was also present but no manual responses were made to it, and it was not correlated with any other stream of information. In the second experiment, two concurrent streams of location-based stimuli were presented. Both were sequenced but only one sequence required responses. Importantly, the sequences were either correlated with one another or not (same vs. different lengths). In the third experiment, the same design was used but with one sequence visual and the other auditory. In all three experiments, participants became sensitive to the sequence that required responses, and resultant knowledge was largely explicit. They were also sensitive to the sequence that did not require responses but only when it was correlated with the sequence that did, and here resultant knowledge was implicit. The findings suggest that the presence of a secondary sequence can affect learning, but only when stimuli in that sequence are integrated, through correlation, with responses made to the primary sequence.
\end{abstract}

J. Cock and B. Meier contributed equally to this work.

J. Cock · B. Meier $(\bowtie)$

Department of Psychology, University of Bern,

Muesmattstr. 45, 3000 Bern 9, Switzerland

e-mail: beat.meier@psy.unibe.ch

\section{Introduction}

In the serial reaction time task (SRTT, cf., Nissen \& Bullemer, 1987), as commonly used to investigate sequence learning, a single visual stimulus is presented at one of several on-screen locations and the participant makes a designated key-press response. On subsequent trials, the stimulus is presented at a series of different locations, each necessitating a corresponding key-press response. This stimulus-response sequence cycles repeatedly throughout a number of blocks of trials. With training, response times (RTs) decrease, but when randomly ordered stimuli are presented, RTs increase. They decrease again when the sequence is reinstated, and RT changes are taken as indicative of sequence learning. Using this arrangement, at least two correlated sequences exist, namely, one comprising the order of stimulus locations and the other comprising the order of key-press responses, these being typically of the same length and usually the same structure. Furthermore, there is visuospatial-motor correspondence at the level of the stimuli (flashes or asterisks together with eye movements), and visuospatial-motor correspondence at the level of the responses (keyboard layout together with finger movements). Secondary streams of stimuli can be included in the SRTT to test the parameters of sequence learning (see Riedel \& Burton, 2006; Schmidtke \& Heuer, 1997, amongst others). Similarly, the exact response requirements can be manipulated (see Deroost \& Soetens, 2006; Nemeth et al., 2009; amongst others). The aim of the present work was to explore the relation between correlated streams of information and response relevance in SRTTtype sequence learning.

In this context, we define a stream of information as a temporal flow of data comprising separable successive events that occur in a dynamic learning environment. 
The events can include stimuli, stimulus features (e.g., colour, shape, size, location, identity, etc.), responses, stimulus-response intervals, cues, etc., and any of the streams can occur in random or sequenced order (Meier \& Cock, 2010, 2012; Weiermann, Cock \& Meier et al., 2010). We use the term "correlated streams" in the sense of co-occurrence or correspondence of the sequences (not to be confused with correlation coefficients as used in statistical tests in which the distribution and magnitude of scores of one variable are compared with those of another variable). We specifically refer to the integration of all possible S-S, R-R, S-R, and R-S relations. As such, elements of correlated streams of information can be parsed together according to their crosswise as well as lengthwise associations and the repeated integrative processing of these compound stimuli and responses allows performance to be improved over time (accuracy, speed, retention, etc.). Hence, correlated sequences result in predictable orders of events (e.g., stimuli and responses) that are embedded in the streams of information that are processed and that have underlying mathematical relations that concord well with one another (i.e., same length although not necessarily same structure). Importantly, when the nature or order of any of the events (stimuli or responses) is changed, the overall pattern of relations (i.e., the correlation) is disturbed and the statistical consistency is lost. Hoffmann and Koch (1998) discussed sequence learning of this kind in terms of uncertainty reduction. They pointed out that "relations between successive stimuli (S-S), between successive responses (R-R), between successive stimulus response mappings ( $\mathrm{S}-\mathrm{R}$ ), and between responses and their outcomes (R-S) may all cause redundancy" (p. 192). Similarly, we suggest that what is learned is a series of configurations of information that span across the different streams at any one moment. Specifically, with two (or more) correlated streams of information, a very strong underlying structure emerges, giving rise to greater statistical predictability.

For example, Shin and Ivry (2002) reported an SRTT study involving two sequences of stimuli, one spatial and one temporal, but with responses made only to the former. In one experiment, the response-irrelevant temporal sequence was defined by response-to-stimulus intervals and in the other, by stimulus onset asynchronies. Learning effects were found for the spatial sequence to which responses were made (both experiments) regardless of whether the two sequences of stimuli were correlated or not. In contrast, sequence learning effects were only found for the response-irrelevant temporal sequence (both experiments) when it was correlated with the spatial sequence. Shin and Ivry concluded that, through correlation, timing of event presentation was integrated into the overall mental representation of the task.
Riedel and Burton (2006) also reported an SRTT-type study with two sequences. Participants were presented with a stream of spoken colour words, in which the order of speaker identity (voices of four actors) followed one sequence and the order of colour words followed another. Key press responses were made to either sequence (between subjects), with participants instructed to "ignore" the other dimension. Sequence learning was found in both groups but only for the sequence to which direct responses were made. Simply listening to the other sequence at the same time had no effect. Riedel and Burton concluded that non-intentional learning of an auditory sequence proceeds in a similar way to visual sequence learning and that, in both cases, stimuli need to be tied to responses. Importantly, in that study, the sequences were concurrent but uncorrelated (eight vs. nine elements in length).

It would seem, therefore, that correlation certainly plays a role in sequence learning in the SRTT. However, the results of various studies in the literature suggest that response relevance might be the driving force. By response relevance, we refer to information that is present in the materials (or procedure) that participants are obliged to process, either directly and deliberately as part of experimental task instructions, or indirectly and unintentionally as part of their exposure to other information that is itself response related. It follows that only information that is related to the responses that are made, directly or otherwise, might be incorporated into what is learned.

However, the presence of correlated streams of information is not always obvious. Mayr (1996), for example, ran an SRTT-type experiment with two simultaneous stimulus sequences (visuo-spatial and non-spatial) instead of the usual one (visuo-spatial) and with key press responses made to only one stream. As participants were found to be sensitive to both sequences, which were of different lengths (i.e., uncorrelated), correlation between the stimulus sequences was apparently not necessary for learning to occur in each stream. Even so, one sequence (symbol identities) was linked to key press responses and the other (symbol locations) to eye movements, such that two separate sets of correlated streams of information would seem to have existed.

Experiment 1 of the present study is an extension of Mayr (1996, Experiment 1). Two sequenced but uncorrelated streams of information were presented simultaneously. Responses were made to stimuli in one stream ("attended") but not the other ("ignored"). Response relevance was, therefore, defined in terms of task instructions. As no mention was made of either sequence, any learning that occurred would be incidental, that is, implicit rather than explicit, in the sense that participants did not set out to deliberately uncover the sequences. Even so, resultant knowledge of either or both sequences might become 
explicit, but this aspect of the experiment was not the main focus of interest. We were concerned with the role of response-relevance and the existence of correlated streams of information. Specifically, we hypothesized that we would find sensitivity to the location sequence but not the symbol identity sequence because, in our experiment, the former was correlated with the motor responses, whereas the latter was not.

The critical difference between our Experiment 1 and that of Mayr is that our participants responded to the sequence that Mayr's participants "ignored", and vice versa. Experiments 2 and 3 had a similar arrangement, with response-relevant and response-irrelevant streams of stimuli, but uncorrelated as well as correlated sequences were also tested. In Experiment 2, the stimuli were in the same modality (i.e., both streams are visual) and in Experiment 3 , they were in different modalities (i.e., one stream is visual and the other is auditory). We hypothesized that if correlated streams of information play a crucial role in this kind of sequence learning, then only conditions with this arrangement would show sensitivity effects for an "ignored" secondary sequence. Because the primary sequence was always correlated with the required response sequence, we expected consistent learning effects for the "attended" primary sequence in all three experiments.

\section{Experiment 1}

In the study by Mayr (1996, Experiment 1), participants responded to graphic symbols with the symbols presented at different locations. Importantly, the sequences were of different lengths. Participants acquired sensitivity to both sequences, leading Mayr and others to the conclusion that two simultaneously presented, but otherwise unrelated, stimulus sequences can be learned in this way. The results were attributed to two independent forms of implicit sequence learning, namely, non-spatial and spatial. However, as acknowledged by Mayr, the symbol identity sequence was correlated with the order of key presses (i.e., response-relevant as well as visual because of the key press requirement), while the location sequence was, very likely, separately correlated with the unrecorded order of eye movements (i.e., response-relevant as well as spatial because of the need to look at each stimulus before identifying it). Using the reverse arrangement to Mayr, participants in our Experiment 1 were required to respond to stimulus locations whilst "ignoring" stimulus identities (graphic symbol sequence). If each sequence needs to be response-relevant, we should find sensitivity to the location sequence only, because the location sequence is now correlated with the key presses (as well as putative eye movements), but the symbol sequence is not.
Method

\section{Participants}

Participants were 40 student volunteers ( 12 women), with a mean age of 29 years $(\mathrm{SD}=7)$.

\section{Materials}

Stimuli were presented at four locations forming the corners of an imaginary square with a side length of $22 \mathrm{~cm}$ (visual angle $21^{\circ}$ ). Locations were marked by small squares with side lengths of $1.2 \mathrm{~cm}$. The four symbols were black square, white square, black circle, and white circle. Symbol width and height were $0.4 \mathrm{~cm}$ each. Participants sat at a viewing distance of approximately $60 \mathrm{~cm}$. Responses to where the symbols appeared were entered using four keys on a standard computer keyboard $(\mathrm{v}, \mathrm{b}, \mathrm{n}, \mathrm{m})$ pressed by the left and right index and middle fingers, respectively. The first key from the left was for the upper left location, the second for the upper right, the third for the lower left, and the fourth for the lower right. The experiment, which was run on a laptop computer with a standard keyboard and a 15-inch monitor, was programmed in E-Prime 1.2 (Psychology Software Tools, Pittsburgh, PA, USA).

\section{Procedure}

The procedure was adapted from Mayr (1996, Experiment 1). The essential difference was that participants were instructed to "attend" (i.e., respond) to stimulus locations whilst "ignoring" (i.e., not responding to) stimulus identities. Mayr's participants did the opposite. Hence, our participants did not have to categorize the graphic symbols in any way-they had only to acknowledge that a stimulus, of whatever kind, was at a particular location on any given trial. Participants were tested individually and were told that the experiment concerned speed of reaction time. Instructions emphasized speed and accuracy. The existence of sequences was not mentioned. For each trial, the stimulus remained on screen until a key was pressed. The response to stimulus interval was $500 \mathrm{~ms}$, as used by Mayr (1996).

Participants first practised the location to key-press mappings in a block of 100 trials in which locations and symbol identities followed separate pseudo-random orders, with no successive repetitions. Then they carried out 16 experimental blocks of trials. In blocks in which the order of locations and/or symbols was sequenced, the first four trials were always random. Two sequences were used: DBDABCAC (8 elements) and CDADBCABA (9 elements). The letters refer to the four screen corners, starting with A for upper left an d going clockwise round the 
screen, and/or to the four symbols: $\mathrm{A}=$ black square, $\mathrm{B}=$ white square, $\mathrm{C}=$ black circle, $\mathrm{D}=$ white circle. One of the sequences was applied to the locations and the other to the symbols, with the arrangement counterbalanced across participants. After the initial four random trials, the two sequences cycled simultaneously through 72 trials in each block. For half of the participants, the location sequence was changed to pseudo-random at block 9 (and back to the same sequence as before at block 10) and the symbol sequence was changed to random at block 12 (and back to the same sequence as before at block 13). For the other half, the arrangement was the reverse. Both sequences were changed to random for all participants at block 15 (and back to the same two sequences as before at block 16). Whenever the "attended" sequence changed to random, the order of required responses also changed. There was no correspondence between particular key presses and particular symbol identities. All other aspects of the procedure remained constant.

At the end of the experiment, participants' awareness and explicit sequence knowledge were assessed in the same way as described in Mayr (1996, Experiment 1). First, participants were misinformed that they had been randomly assigned to one of four categories. In the imaginary "group 1 ", both orders (symbols and locations) were said to be "regular"; in "group 2", the symbol order was said to be "random" and the location order "regular"; in "group 3", it was vice versa; and in group 4 , both orders were said to be "random". Participants were asked to indicate to which group they thought they belonged. In fact, all participants had been in "group 1" because both orders had been sequenced, albeit separately (the symbol sequence and the location sequence being of different lengths and hence uncorrelated). Next, they were debriefed about both orders being sequenced and were asked to report (recall) them from memory or by guessing. Finally, by pressing keys, participants were asked to generate one sequence cycle each of what they thought the location and symbol sequences might be. They were given the two initial elements of each sequence and told to predict the rest. For the location sequence, participants entered responses themselves. For the symbol sequence (to which they had not responded previously), participants pointed to the symbols and the experimenter entered the information. The order of presentation of the tasks was counterbalanced. The maximal numbers of consecutively correct sequence elements were calculated.

\section{Data analysis}

Error rates and median response times per block and per participant were computed and averaged. As no counterbalancing effects (order of sequence change to random, block 9 or block 12, and sequence length, eight or nine elements) were found, these are not included in the results section below. For the analysis of sequence learning effects, we compared RT differences between pseudorandom and sequenced blocks of trials for the two types of stimulus sequence (symbol location sequence with key press responses vs. symbol identity sequence with no key press responses). The dependent variable was RT measured in milliseconds. Disruption scores were calculated as the RT differences for pseudo-random blocks compared with surrounding sequenced blocks. For half the participants, the location sequence was changed to random at block 9 and the symbol sequence was changed to random at block 12 . For the other half it was the reverse. The order of the sequences ( 8 or 9 elements) was also counterbalanced. At block 15 both sequences were changed to random at the same time.

\section{Results}

\section{Error rates}

Across all experimental blocks and all participants, the mean error rate was close to floor level $(M=0.04$, $\mathrm{SD}=0.03$ ) and was not analysed further.

Disruption scores were calculated separately for the location sequence to which key press responses were made and the symbol sequence to which no responses were made (RT differences between random and surrounding blocks; see Fig. 1). For the response-relevant location sequence, a $t$ test revealed a sequence learning effect in the form of a significant increase in RTs in the random blocks (blocks 9 and 12 combined), $M=95 \mathrm{~ms}(\mathrm{SD}=77), t(39)=7.81$, $p<0.001$. For the response-irrelevant symbol sequence, a decrease rather than an increase in RTs was found, $M=$ $-12 \mathrm{~ms}(\mathrm{SD}=38), t(39)=-1.96, p=0.06$ (see Table 1$)$.

At block 15, where both sequences were changed to random together, the overall mean disruption score was $123 \mathrm{~ms}$ (SD =98), with an RT comparison to surrounding blocks giving $t(39)=7.92, p<0.01$. Participants for whom the location sequence was changed to random at block 9 and the symbol sequence was changed to random at block 12 showed a disruption score of $143 \mathrm{~ms}(\mathrm{SD}=112)$. Participants for whom the symbol sequence was changed to random at block 9 and the location sequence was changed to random at block 12 showed a disruption score of $103 \mathrm{~ms}$ $(\mathrm{SD}=78)$. An independent samples $t$ test showed no difference between them, $t(38)=1.33, p=0.19$.

\section{Explicit knowledge}

An initial Chi-squared test on the pattern of responses to four hypothetical categories (locations and symbols in random order, both sequenced, only one or the other 


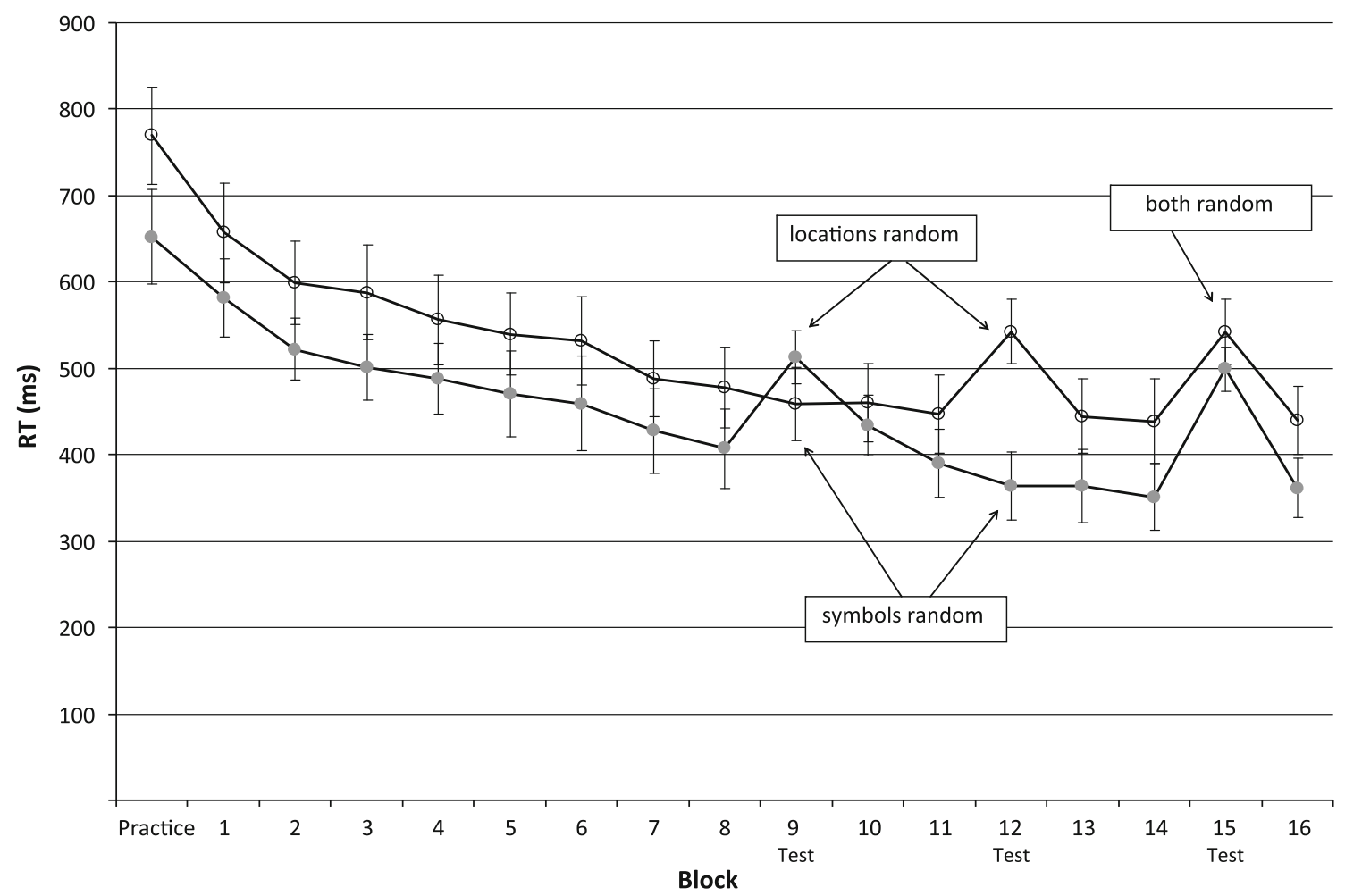

Fig. 1 Experiment 1: response times in $\mathrm{ms}$ as a function of blocks, shown separately for participants with a random order of locations in block 9 and a random order of symbols in block 12 (black circles on graph) compared with those with the reverse (white circles on graph).
Both orders were random in block 15. Error bars represent standard errors. All participants made key press responses to the order of stimulus locations. The symbol sequence and the location sequence were uncorrelated

Table 1 Mean disruption scores calculated in ms as RT differences between pseudo-random blocks and surrounding sequenced blocks

\begin{tabular}{|c|c|c|c|c|c|}
\hline \multirow[t]{2}{*}{ Experiment } & \multirow[t]{2}{*}{ Required responses } & \multicolumn{2}{|c|}{ Correlated condition changed to random } & \multicolumn{2}{|c|}{ Uncorrelated condition changed to random } \\
\hline & & "attended" & "ignored" & "attended" & "ignored" \\
\hline 1 & Locations & - & - & $95 * *$ & -12 \\
\hline 2 & Asterisks & $132 * *$ & $25^{*}$ & $82 * *$ & -5 \\
\hline 3 & Asterisks & $121 * *$ & $17 *$ & $70 * *$ & -5 \\
\hline 3 & Tones & $234 * *$ & $60 * *$ & $164 * *$ & -0 \\
\hline
\end{tabular}

Experiment 1: two sequences, locations of symbols (response-relevant) and identity of symbols (response-irrelevant). Experiment 2: two sequences of asterisks both visuo-spatial (location-based). Experiment 3: same sequences as in Experiment 2 but one visuo-spatial and the other auditory

* Significantly different from zero, $p<0.05$, ** Significantly different from zero, $p<0.001$

sequenced) revealed no significant difference $(p>0.1)$, suggesting that participants' self-assignment was largely uninformed. According to Mayr (1996, Experiment 1), the level for reproducing an eight- or nine-element sequence purely by chance (first and 2nd element provided) is $33.3 \%$. Six participants, who (correctly) suspected that both orders were probably sequenced, correctly recalled $73 \%$ of the location sequence ( $p<0.05$, single-sample $t$ test against chance) and correctly generated $76 \%$ of it ( $p<0.05$, single-sample $t$ test against chance). They correctly recalled only $28 \%$ of the symbol sequence and generated $29 \%$. Sixteen participants, who suspected that only the location stream was sequenced, correctly recalled $56 \%$ of it $(p<0.05)$ and correctly generated $68 \%$ $(p<0.05)$. By guessing, they correctly reported $31 \%$ of the symbol sequence and generated $34 \%$. Eight participants, who suspected that only the symbol order was sequenced, correctly reported $28 \%$ of it and generated $25 \%$. By guessing, they correctly reported only $26 \%$ of the location sequence but generated $49 \%(p<0.05)$. The remaining ten participants, who suspected that both orders were random, correctly reported only $18 \%$ of the location 
sequence but correctly generated $54 \%$ of it $(p<0.05)$. They correctly reported $21 \%$ of the symbol sequence and generated $26 \%$. Thus, participants had reliable explicit knowledge of the response-relevant sequence (locations) but not the response-irrelevant sequence (symbols).

\section{Discussion}

The aim of Experiment 1 was to test for sequence learning using two unrelated stimulus sequences, only one of which was directly response-relevant according to the task instructions. Significant sequence learning effects, in terms of changes in response times, were found for the location sequence to which participants made key press responses. In contrast, no learning effects were found for the symbol identity sequence to which no responses were required to be made. Hence, we found no support for the idea that purely visual ("perceptual") sequence learning of a secondary sequence can occur. Rather, it seems that responserelevance is necessary.

The implication would be that Mayr (1996) found learning of an "ignored" symbol location sequence (using the reverse arrangement to ours) because it was responserelevant. In order to respond to what the symbols were (identity sequence accompanied by manual responses), Mayr's participants had to look at where the symbols were (location sequence accompanied by eye movement responses), even though the two sequences were of different lengths (i.e., uncorrelated with one another). Indeed, Mayr himself noted that, "both orienting of attention (or eye movements) and selection of a motor output can be considered as responses" (p. 360). In his experiment, the motor sequence of eye movements would have correlated perfectly with the visuo-spatial sequence of locations at which the symbols appeared. We suggest, therefore, that correlation, together with response-relevance, can account for sensitivity towards the "ignored" sequence in Mayr's experiment. In contrast, in Experiment 1 of the present study, it was not necessary for participants to categorize the symbols to respond to where they were. They just needed to see that a stimulus, of whatever kind, was present. In fact, we did not want participants to categorize the symbols in any way (i.e., before responding to their locations) because this might have set up a separate order of covert responses (i.e., in addition to the overt motor key press responses). An additional hidden sequence of this kind, had it existed, would have correlated with the symbol identity sequence.

It could also be argued that in Experiment 1, we found a difference in the incidental learning of a spatial versus a non-spatial sequence simply because they are different in nature (see Koch \& Hoffmann, 2000). However, although we found learning of the spatial sequence but not the nonspatial, Mayr found learning of both, using exactly the same sequences (1996, Experiment 1). We suspect, therefore, that the difference lies in the design and instructions and not the materials. It would seem that sequence correlation can facilitate the learning of a secondary sequence, whereas a lack of correlation can impede it. Hence, sequence correlation was the focus of Experiment 2.

\section{Experiment 2}

In Experiment 2, participants were exposed to two visualspatial streams at once, with one hidden sequence being response-relevant and the other, technically speaking at least, response-irrelevant (cf., Cock, Berry \& Buchner, 2002). The stimuli were presented simultaneously and the sequences were both location-based, with different colours used to separate the streams. The response-irrelevant sequence was either correlated or not with the responserelevant sequence by means of same versus different length sequences. We predicted that participants would become sensitive to the response-relevant sequence in both conditions and perhaps to the response-irrelevant sequence as well-but, in this case, only in the correlated sequences condition. This is because when the sequences of stimuli were uncorrelated (for example, 6 vs. 7 elements long), there were no predictable associations between the stimulus locations in the two streams (at least not over 42 trials) or between the second sequence and the key press responses. In contrast, when the sequences of stimuli were correlated (both six or both seven elements long), predictable associations could give rise to the formation of compound stimuli through integrative processing, such that what appeared to be irrelevant information might actually become relevant and thereby facilitate sensitivity to both sequences.

\section{Method}

\section{Participants and design}

Participants were 40 undergraduate student volunteers (19 women), with a mean age of 23 years $(\mathrm{SD}=7)$. They were assigned to one of two conditions, correlated sequences (two asterisks) and uncorrelated sequences (two asterisks). As in Experiment 1, RTs in pseudo-random blocks of trials were used to test for sequence learning effects (disruption scores). For the main analysis, we used a mixed design, with sequencing (correlated vs. uncorrelated) manipulated between subjects and block (sequenced vs. pseudo-random) manipulated within subjects. 


\section{Materials}

Each stimulus display comprised two $0.5 \times 0.5 \mathrm{~cm}$ asterisks, one coloured red and the other blue ("attended" and "ignored" according to counterbalanced instructions). They were presented simultaneously, against a pale grey background, at two out of four horizontally aligned locations. The locations were positioned $8 \mathrm{~cm}$ above the bottom of the screen and marked by underline bars each $3 \mathrm{~cm}$ apart. The different coloured asterisks used the same locations but never appeared simultaneously at the same location (in such an event, the computer program automatically changed the location of the "ignored" asterisk to a different location at random for that particular trial only). Four hybrid sequences (i.e., with ambiguous as well as unique transitions) were used in a counterbalanced way, 123243 (6 elements), 241321 (6 elements), 1423243 (7 elements), and 2341321 ( 7 elements), where the numbers correspond to the horizontally aligned asterisk locations from left to right, as well as to the layout of the response keys. The arrangement of the four keys ( $v, b, n, m)$ was such that responses were always consistent and spatially compatible with the four on-screen "attended" asterisks locations. As in Experiment 1, the experiment was run on a laptop computer with a standard keyboard and a 15-inch monitor.

\section{Procedure}

The procedure was similar to Experiment 1. Participants were instructed to press one of four designated keys in response to whichever of the locations was occupied by the to-be-attended asterisk (red or blue, as instructed). On each trial, the two asterisks remained on screen until the participant pressed the appropriate key. The response to stimulus interval was $400 \mathrm{~ms}$. The existence of sequences was not mentioned until the end of the experiment. All participants first practised the task in two blocks of 84 trials with a pseudorandom order of locations. They performed 13 blocks of trials in total (84 trials per block, with an additional single random trial at the beginning of each block). Blocks 1-6, 9 and 10, and 13, were sequenced (both streams, correlated or uncorrelated according to sequence length). In blocks 7 and 8, the order of locations of the response-irrelevant asterisk was changed to pseudorandom while the order of locations of the response-relevant asterisk remained sequenced. In blocks 11 and 12, the order of locations of the response-relevant asterisk was changed to random while the order of locations of the responseirrelevant asterisk remained sequenced. Throughout the experiment, whenever the response-relevant sequence was changed to random, so was the required response order, but whenever the response-irrelevant sequence was changed to random, the required response order remained unchanged. All other aspects of the procedure remained constant throughout, including the critical blocks. After the final block, participants were questioned about the order of asterisk locations and the possible existence of sequences.

\section{Data analysis}

Similar measures and constraints were used as in Experiment 1. As a preliminary analysis of RT data showed no adverse counterbalancing effect of sequence length or of asterisk colour, these variables were not included in the analyses reported here.

Results

\section{Error rates}

The mean error rate was low: $\mathrm{M}=0.06(\mathrm{SD}=0.05)$ averaged across sequenced blocks 1-10 and block 13, and $\mathrm{M}=0.09(\mathrm{SD}=0.07)$ averaged across random blocks 11 and 12. Error data were not analyzed further.

\section{Blocks 6-9}

Sensitivity to the sequence of response-irrelevant asterisk locations was examined by changing this sequence to pseudorandom at blocks 7 and 8. Mean RTs, based on individual mean RTs, were averaged across blocks 7 and 8 and compared with the average of surrounding blocks 6 and 9. Using a $2 \times 2$ mixed ANOVA, a significant main effect of block was found, with $F(1,38)=8.30$, MSE $=237, p<0.01$, showing disruption of responses at blocks 7-8. Despite no main effect of sequencing (correlated sequences vs. uncorrelated sequences), there was a significant block $x$ sequencing interaction, $F(1,38)=18.89, \quad \mathrm{MSE}=237, p<0.001$, reflecting the fact that whilst participants in the correlated sequences condition were disrupted, those in the uncorrelated sequences condition were not (see Fig. 2). For blocks 7-8, the mean disruption scores were $25 \mathrm{~ms}(\mathrm{SE}=5)$ for the correlated sequences condition, with a single sample $t$ test against zero giving $t(19)=4.79, p<0.001$, and $-5 \mathrm{~ms}(\mathrm{SE}=4.5)$ for the uncorrelated sequences condition, $t(19)=1.12$, $p=0.28$.

\section{Blocks 10-13}

Sensitivity to the sequence of response-relevant asterisk locations was examined by changing this sequence to pseudorandom at blocks 11-12. Mean RTs, based on individual mean RTs, were averaged across blocks 11 and 12 and compared with the average of surrounding blocks 10 and 13 . Using a $2 \times 2$ mixed ANOVA, a significant main effect of 


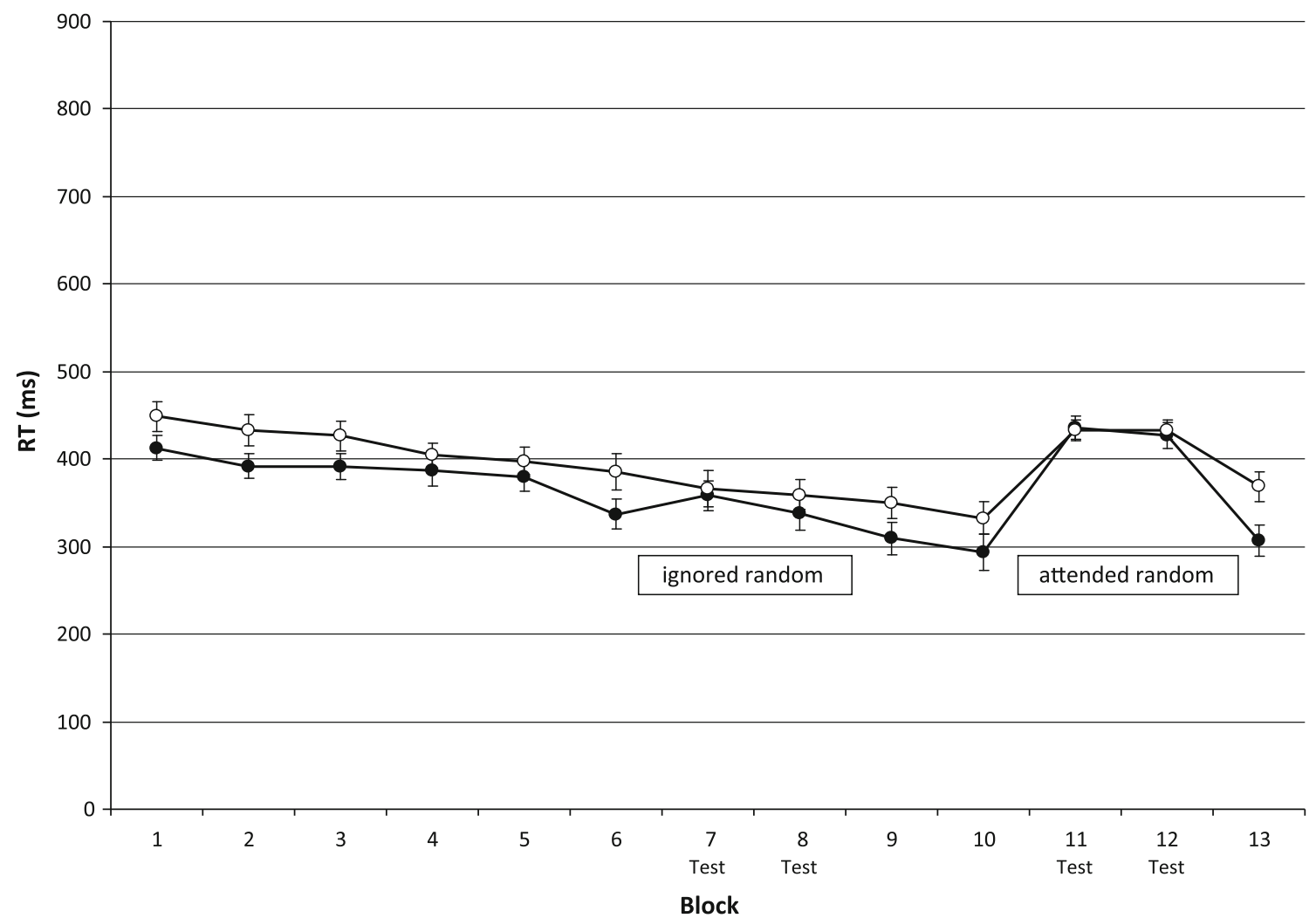

Fig. 2 Experiment 2: response times in ms as a function of blocks, shown separately for participants with a correlated secondary asterisk sequence (black symbols on graph) and for those with an uncorrelated secondary asterisk sequence (white symbols on graph). For the experimental groups, the order of "ignored" asterisks was random in

block was found, with $F(1,38)=122.08, \operatorname{MSE}=1,869$ $p=<0.001$, showing disruption of responses at blocks 11-12. Despite no main effect of sequencing, there was a significant block $x$ sequencing interaction, with $F(1,38)=$ $6.55, \operatorname{MSE}=1,869, p=0.015$, reflecting the fact that whilst participants in both conditions were disrupted, those in the correlated condition were more disrupted than those in the uncorrelated condition (see Fig. 2). For blocks 11-12, the mean disruption scores were $132 \mathrm{~ms}(\mathrm{SE}=15)$ for the correlated sequences condition, with a single sample $t$ test against zero giving $t(19)=8.85, p<0.001$, and $82 \mathrm{~ms}$ $(\mathrm{SE}=12)$ for the uncorrelated sequences condition, $t(19)=6.64, p<0.001$.

\section{Explicit knowledge}

Participants in both conditions were generally aware that the order of asterisk locations, to which they made responses, was sequenced. They could report nearly all of this sequence by pointing at the locations on screen and generating the order of key press responses (i.e., 6 or 7 elements). Most participants were aware that the order of these response-relevant locations changed in some way in blocks 7 and 8 and the order of "attended" asterisks was random in blocks 11 and 12. Error bars represent standard errors. All participants made responses to the order of asterisk locations (primary sequence, "attended"). The other stream of asterisk locations was response-irrelevant (secondary sequence, "ignored")

the second half of the experiment, but reverted to the same sequence later on. Five out of twenty participants $(25 \%)$ in the correlated sequences condition and three out of twenty $(15 \%)$ in the uncorrelated sequences conditions thought that the order of asterisks locations to which no responses had been made might also have been sequenced, but none felt able to report that sequence (also 6 or 7 elements in length). Thus, participants appeared to have verbalizable explicit knowledge of the response-relevant sequence, but not the response-irrelevant sequence.

\section{Discussion}

The aim of Experiment 2 was to investigate sequence learning and response relevance vis-a-vis correlated and uncorrelated sequences. Two sequences were presented simultaneously, with one stream of stimuli termed response-relevant ("attended") and the other responseirrelevant ("ignored"). First, in the correlated sequences condition, participants were found to be sensitive to both sequences, but in the uncorrelated sequences condition, they were sensitive to response-relevant sequence only. Second, whereas participants became aware of the presence 
of the response-relevant sequence and acquired explicit knowledge of it, they remained largely unaware of the response-irrelevant sequence, irrespective of whether the two sequences were correlated or not. Third, sequence learning effects for the response-relevant sequence were greater in the correlated sequences condition. We conclude that participants can become sensitive to a sequence of supposedly response-irrelevant stimuli, but only when that sequence is integrated into the response-relevant information, in which case its status is changed. That is, supposedly response-irrelevant stimuli may actually become responserelevant through correlation between the sequences. Furthermore, the integration would have been broken when either sequence was changed to random (see Keele, Ivry, Mayr, Hazeltine, \& Heuer, 2003; Schmidtke \& Heuer, 1997; Schwarb \& Schumacher, 2010). From the size of the disruption scores, we conclude that correlation between sequences strengthens the main sequence learning. When taken together, the results suggest that response-relevance was driving the sequence learning-but correlation between the sequences provided the mechanism (cf. Cock \& Meier, 2007; Meier \& Cock, 2010, 2012; Weiermann et al., 2010; Weiermann \& Meier, 2012).

In the uncorrelated sequences condition, the responserelevant primary sequence was still learned even when the response-irrelevant sequence was not. This is not surprising because the primary sequence was correlated with the key press response order as well as with the visuo-spatial arrangement on the keyboard.

\section{Experiment 3}

In Experiment 2, the two stimulus sequences were presented in the same modality. The aim of Experiment 3 was to establish if the same pattern of results could be found with stimuli in different modalities. We reasoned that using two modalities might make integration of correlated sequences more difficult. In this way, sensitivity to a secondary sequence might be "discouraged" through greater efficiency at properly ignoring it, even when correlated with the primary sequence. In order to test this, we combined a sequence of asterisk locations (visuo-spatial) with a sequence of tones of different pitches (auditory).

Method

\section{Participants and design}

Participants were 80 undergraduate student volunteers (58 women), with a mean age of 23 years $(\mathrm{SD}=4)$. They were assigned to one of four conditions at random: correlated sequences: respond to tones (and "ignore" asterisks), correlated sequences: respond to asterisks (and "ignore" tones), uncorrelated sequences: respond to tones (and "ignore asterisks"), uncorrelated sequences: respond to asterisks (and "ignore" tones). As in Experiments 1 and 2, RTs in pseudorandom blocks of trials were used to test for sequence learning effects (disruption scores). Sequencing (correlated vs. uncorrelated) and presentation (attend tones and ignore asterisks vs. attend asterisks and ignore tones) were manipulated between subjects, whilst block was manipulated within subjects, resulting in a mixed design. As in Experiment 2, the experimental conditions comprised 13 blocks of trials.

\section{Materials and apparatus}

These were as in Experiment 2 except that only one stream of asterisks was presented in the experimental conditions. The other stream comprised a series of four tones, namely, 300, 500, 700 and $900 \mathrm{~Hz}$, which, for participants who were required to respond to tones (rather than asterisks), were mapped to the keys $\mathrm{v}, \mathrm{b}, \mathrm{n}$, and $\mathrm{m}$, respectively. The tones were delivered by an external loudspeaker attached centrally to the top of the computer monitor. Tones and asterisks were presented simultaneously as concurrent events and the same versus different length sequences arrangement was used in a counterbalanced way as in Experiment 2. The sequences themselves were also as in Experiment 2, with the visual sequence determined by the order of asterisk locations, and the tone sequence determined by order of the tones.

\section{Procedure}

For participants who were required to respond to asterisks, the procedure was as in Experiment 2, but with the secondary stream comprising tones that had to be "ignored" instead of other asterisks. For participants who were required to respond to tones, the procedure was the same, but with participants simultaneously "ignoring" a secondary stream of asterisk locations. The same response keys were used as in Experiment 2. On each trial, the tone and the asterisk remained present until the appropriate key was pressed. In order to ensure that participants who responded to tones still looked at the screen and saw the asterisks, all participants were misinformed that "The screen might change colour at some point, please take note if this occurs and remember to report it at the end of the experiment". The post task interview was adapted to include questions about tones as well as asterisks.

\section{Data analysis}

The same measures, constraints, and analyses were used as in Experiment 2, with allowance being made for the 
inclusion of tones as well as asterisks and the presentation of only one asterisk instead of two.

Results

\section{Error rates}

For participants who responded to tones, mean rates were $M=0.11(\mathrm{SD}=0.08)$ in sequenced blocks $1-10$ and block 13 and $M=0.17(\mathrm{SD}=0.09)$ in random blocks 11 and 12 . For participants who responded to asterisk locations, they were $M=0.06(\mathrm{SD}=0.05)$ in sequenced blocks $1-10$ and in block 13 and $M=0.10$ (SD =0.07) in random blocks 11 and 12. Error data were not analyzed further.

\section{Blocks 6-9}

Sensitivity to the response-irrelevant sequence (tones or asterisks) was examined by changing only this sequence to pseudorandom at blocks 7 and 8. Mean RTs, based on individual mean RTs, were averaged across blocks 7 and 8 and compared with the average of surrounding blocks 6 and 9. Using a $2 \times 2 \times 2$ mixed ANOVA, a significant main effect of block was found, $F(1,76)=9.49$, MSE $=1,013, p<0.001$, showing disruption of responses at blocks 7-8. Despite no main effect of sequencing, there was a significant block $x$ sequencing interaction, $F(1,76)=20.57, \mathrm{MSE}=1,013, p<0.001$, showing that whereas participants in the correlated conditions were disrupted when the response-irrelevant sequence was changed to random, those in the uncorrelated conditions were not (see Fig. 3). There was a significant main effect of presentation, $F(1,76)=33.38, \quad \mathrm{MSE}=71,669$, $p<0.001$, showing that participants who responded to tones were generally slower than participants who responded to asterisks, together with a significant block $x$ sequencing $x$ presentation interaction $F(1,76)=5.71$, MSE $=1,013, p=0.02$, showing that disruption of RTs in the correlated conditions was greater for participants who responded to tones than for those who responded to asterisks. No other interaction reached significance.

For blocks 7-8, for participants who responded to tones, the mean disruption scores were $60 \mathrm{~ms}(\mathrm{SE}=17)$ for the correlated sequences condition, with a single sample $t$ test against zero giving $t(19)=3.51, p=0.05$, and $-10 \mathrm{~ms}$ $(\mathrm{SE}=8)$ for the uncorrelated sequences condition, $t(19)=1.28, p=0.22$. For participants who responded to asterisks, the mean disruption scores were $17 \mathrm{~ms}$ $(\mathrm{SE}=6.5)$ for the correlated sequences condition, with a single sample $t$ test against zero giving $t(19)=2.57$, $p<0.05$, and $-5 \mathrm{~ms}(\mathrm{SE}=3)$ for the uncorrelated sequences condition, $t(19)=1.39, p=0.18$.

\section{Blocks 10-13}

Sensitivity to the sequence of response-relevant asterisk locations was examined by changing only this sequence to pseudorandom at blocks 11 and 12. Mean RTs, based on individual mean RTs, were averaged across blocks 11 and 12 and compared with the average of surrounding blocks 11 and 13. Using a $2 \times 2 \times 2$ mixed ANOVA, a significant within-subjects main effect of block was found, $F(1,76)=203.74$, MSE $=4,255, p<0.001$, reflecting disruption of responses at blocks 11-12. Despite no main effect of sequencing, there was a significant block $x$ sequencing interaction, $F(1,76)=8.62, \mathrm{MSE}=4,255$, $p<0.01$, showing that participants in the correlated conditions were disrupted more than those in the uncorrelated conditions (see Fig. 3). There was a significant main effect of presentation, $F(1,76)=50.32, \quad \mathrm{MSE}=59,631$, $p<0.001$, showing that participants who responded to tones were generally slower than participants who responded to asterisks, together with a block $x$ presentation interaction, $F(1,76)=25.35, \mathrm{MSE}=4,255, p<0.001$, showing that participants who responded to tones were disrupted more than those who responded to asterisks. No other interaction reached significance.

For blocks 11-12, for participants who responded to tones, the mean disruption scores for blocks 11-12 were $234 \mathrm{~ms}(\mathrm{SE}=29)$ for the correlated sequences condition, with a single sample $t$ test against zero giving $t(19)=8.02$, $p<0.001$, and $164 \mathrm{~ms}(\mathrm{SE}=26)$ for the uncorrelated sequences condition, $t(19)=6.41, p<0.001$. For participants who responded to asterisks, the mean disruption scores for blocks 11-12 were $121 \mathrm{~ms}(\mathrm{SE}=11) \mathrm{ms}$ for the correlated sequences condition, with a single sample $t$ test against zero giving $t(19)=11.05, p<0.001$, and $70 \mathrm{~ms}$ $(\mathrm{SE}=9)$ for the uncorrelated sequences condition, $t(19)=8.15, p<0.001$.

\section{Explicit knowledge}

All participants were generally aware that the order of the stimuli to which they made responses (i.e., the "attended" stream of information) was sequenced, asterisk locations and tones alike. As in Experiment 2, they could report most of this sequence. Most participants (correlated and uncorrelated conditions alike) were also aware that the order of the "attended" stimuli changed in the second half of the experiment and reverted to the same sequence later on. Taken together, $36 \%$ of the participants (29 out of a total of 80) thought that the "ignored" stream might also have been sequenced ( 7 correlated sequences condition, respond to tones; 13 correlated sequences condition, respond to asterisks; 4 uncorrelated sequences condition, respond to tones; 5 uncorrelated sequences condition, respond to 


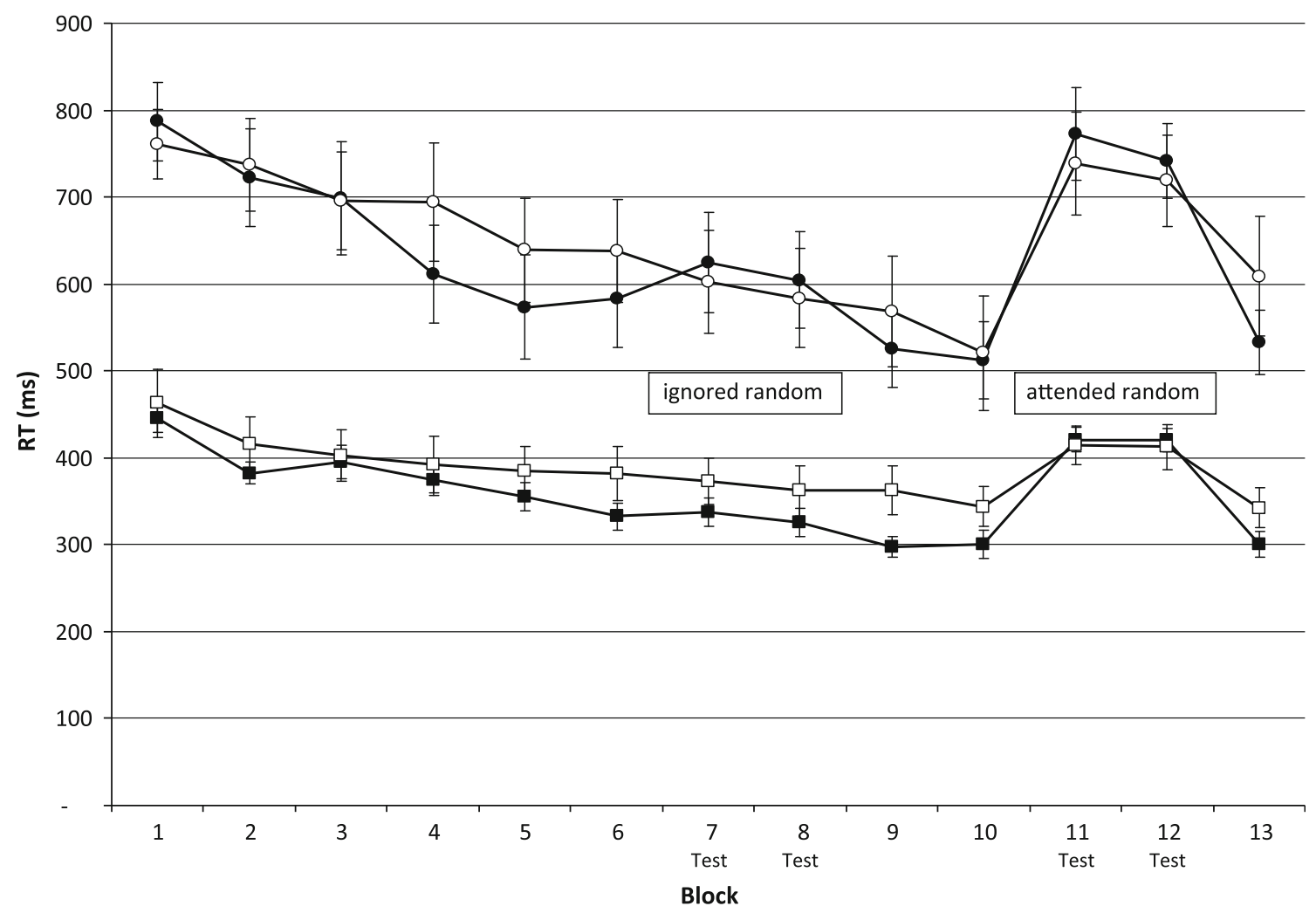

Fig. 3 Experiment 3: response times in $\mathrm{ms}$ as a function of blocks, shown separately for participants with a correlated secondary sequence (black symbols on graph) and those with a uncorrelated secondary sequence (white symbols on graph), as well as separately for those who responded to asterisk locations whilst "ignoring tones" (squares on graph) and those who responded to tones whilst

asterisks). However, none of the 29 felt able to report the "ignored" sequence. Thus, as in the previous experiment, participants appeared to have reliable explicit knowledge of the sequence to which they made responses but not the sequence to which no responses were made.

\section{Discussion}

The aim of Experiment 3 was to replicate the finding of Experiment 2 but with stimuli presented in two modalities. Exactly as in Experiment 2, when the stimulus sequences were correlated, participants were sensitive to both sequences, but when they were not, participants were only sensitive to the sequence to which they made manual responses. This applied equally to "attend tones and ignore asterisks" and "attend asterisks and ignore tones" conditions.

First, as in Experiment 2, we conclude that sequencing in a secondary stream can only be learned about indirectly, that is, when it is integrated into the main response-relevant sequence learning through correlation between the sequences. Second, we conclude that sequence integration "ignoring" asterisk locations (circles on graph). For the experimental groups, the order of response-irrelevant stimuli ("attended") was random in blocks 7 and 8 and the order of response-relevant stimuli ("ignored") was random in blocks 11 and 12. Error bars represent standard errors

operates equally well across as within modalities, and there was no indication that using two modalities made "ignoring" the secondary stimuli easier, or harder. Third, as in Experiment 2, sequence learning was enhanced in the correlated conditions compared with the uncorrelated. Fourth, the results of Experiment 3 showed greater disruption with auditory than visual stimuli as the primary response-relevant dimension (see Table 1). However, rather than suggesting that auditory sequences are learned more readily than visual, it seems to be more the case that, upon disruption of RTs (when the sequence changed to random), participants reverted to responding at the level at which they began the experiment. Pressing keys in response to sounds was less familiar and took longer throughout. This difference in magnitude of disruption scores is therefore a performance effect only.

For this reason, Koch and Hoffmann (2000) have pointed out that the SRTT is particularly well suited to visuospatial stimuli combined with motor-spatial responses. Using an orthogonal design, they tested participants on spatial versus symbolic stimuli (asterisk locations vs. digits) and spatial versus symbolic responses (keystrokes vs. 
verbal digit naming), and found much stronger sequence learning effects with spatial stimuli and/or spatial responses. It means that, when SRTT results are compared between conditions or experiments, the extent of sequence learning should not be equated with the absolute size of the mean disruptions scores (see Hoffmann \& Koch, 1998 for related discussions).

\section{General discussion}

We investigated the role of correlated sequences and response relevance in sequence learning. This was achieved by presenting two concurrent stimulus sequences, one stream directly response-relevant ("attended") and the other supposedly response-irrelevant ("ignored"), and by using sequences of the same or different lengths (correlated vs. uncorrelated). In all three experiments, we found significant learning of the primary sequence, that is, the one that was always correlated with the order of responses. Additionally, we found sensitivity to the secondary sequence, to which no overt responses were made, but only when it was correlated with the primary sequence. We attribute this result to the secondary sequence becoming indirectly response-relevant through the correlation.

Specifically, in Experiment 1, significant sequence learning effects, together with explicit knowledge, were found for a symbol location sequence in response to which participants made correlated key presses and putative eye movements. No learning effects, or explicit knowledge, were found for a concurrent symbol identity sequence to which no responses were made (no other correlated sequence being present). Experiment 2 comprised two concurrent location-based sequences of stimuli, one stream being response-relevant ("attended") and the other supposedly response-irrelevant ("ignored"). When the sequences were correlated, participants showed significant learning effects when either sequence was changed to random. However, when they were not correlated, learning effects pertained only to the sequence to which key press responses were made. Whereas participants became aware of the primary sequence and acquired explicit knowledge of it, they remained unaware of the secondary sequence, whether the two were correlated or not. A very similar pattern of results was found in Experiment 3, in which visual and auditory modalities were used. Again, participants acquired sensitivity to the primary response-relevant sequence, together with explicit awareness of it, irrespective of whether it was correlated with the secondary response-irrelevant sequence or not, but no sensitivity to the secondary sequence unless it was correlated with the primary sequence. This applied to auditory and visual stimuli alike.
Taking the three experiments together, we conclude that, where correlated sequences exist, a compound order of complex stimuli or "configurations" is formed. As a result, a "super-sequence" exists and, through the correlation, "ignored" stimuli are processed and become relevant to what is learned through the responses that are made. Importantly, consistency between elements of a secondary sequence and the responses made to a primary sequence can be highly regular. For example, in Experiments 2 and 3, with the combination 123243 (secondary sequence) and 241321 (primary sequence), when the participant presses the 2nd key (b), the "ignored" stimulus is always at either the 1st or 4th location. When s/he presses the 4th key (m), the "ignored" stimulus is always at the 2nd location, and so forth. This predictable regularity across the streams changes, or is lost, when either sequence is switched to random and can, therefore, contribute to RT disruption effects. It is exactly this kind of integrated statistical structure that gives rise to the "supersequence" mentioned above (see Ziessler and Nattkemper (2001) for comments in terms of S-R-S chaining effects and Abrahamse, Jiménez, Verwey, and Clegg (2010) for related comments in terms of integrated sequence learning).

The implication is that, in Experiments 2 and 3, both sequences of stimuli were processed despite the instruction to "attend" to only one stream. Indeed, it could be argued that only the "super-sequence" was learned under such circumstances. However, there may be limitations on how much information can be incorporated into this kind of integrative sequence learning. Rowland and Shanks (2006), for example, used the SRTT to test the simultaneous learning of multiple contingencies. After extensive training on probabilistic sequences, as expected, participants were found to be sensitive to a primary sequence (location based). They were also sensitive to a statistically different secondary sequence (also location based and of the same length as the primary sequence) but only when the perceptual load of the primary task was low. The authors concluded that there might be attentional restrictions on the learning of multiple contingencies in the SRTT. In order to test the role of attention Jiménez and Mendez (1999) used different shapes as stimuli "place holders", such that the particular shape on the present trial predicted the stimulus location on the next trial. This predictability was found to contribute positively to sequence learning but only when the shapes were selectively (i.e., deliberately) attended. Furthermore, sequence learning was not adversely affected by the inclusion of a secondary counting task. Jiménez and Mendez concluded that sequence learning of this kind is an automatic associative process that needs very little in the way of attentional resources but that selective attention affects what is learned.

It is even possible that, after sufficient practice, a secondary task, such as tone counting in SRTT dual-task studies, can become integrated into the primary task 
(Rah, Reber \& Hsiao, 2000; Hsiao \& Reber, 2001). The degree to which any two tasks can be integrated depends on the exact task requirements and the sequenced or random nature of the sequences. When the stimuli for the tone-counting task follow a same-length sequence as the primary SRTT sequence (i.e., if these sequences are correlated), this gives rise to another more complex, multi-component sequence. As suggested by Rah et al., participants seem to scan the environment for patterns of co-variation automatically and where there is predictability between the streams in different dimensions, conjoint learning is facilitated. In other words, dual-tasking in SRTT-type experiments is not so very different from the combined learning of primary and secondary sequences-both depend on the degree to which processing of different streams of information can be combined.

In the present study, we found that sequence learning effects for a response-relevant sequence were greater when the sequence was accompanied by a correlated responseirrelevant sequence. Similarly, Hoffmann, Sebald, and Stoecker (2001) and Stoecker, Sebald, and Hoffmann (2003) found that if intermittent tones were introduced into an SRTT-type experiment and contingently mapped onto the responses made to the primary sequence, then that sequence learning was enhanced. The improvement occurred despite the fact that the tones were supposedly irrelevant to key presses made in response to visual stimuli and lends support to the idea that correlation between different streams of information strengthens primary sequence learning (see also Robertson, Tormos, Maeda, \& Pascual-Leone, 2001; Schmidtke \& Heuer, 1997; Shin \& Ivry, 2002).

In summary, we have shown that a supposedly responseirrelevant sequence of stimuli can indeed become part of what is learned in an SRTT, but only when it is correlated with a response-relevant sequence, and thereby integrated into the main sequence learning through the responses. The results of all three experiments are consistent with a response-relevant account of incidental sequence learning, but with correlation between the various streams as an essential pre-condition.

Acknowledgments The study was supported by a grant from the Swiss National Science Foundation (Grant 130104) to B. Meier. We thank Brigitte Weiermann for programming and data management in Experiment 1.

\section{References}

Abrahamse, E. L., Jiménez, L., Verwey, W. B., \& Clegg, B. (2010). Representing serial action and perception. Psychonomic Bulletin \& Review, 17, 603-623.

Cock, J. J., Berry, D. C., \& Buchner, A. (2002). Negative priming and sequence learning. European Journal of Cognitive Psychology, $14,27-48$.
Cock, J., \& Meier, B. (2007). Incidental task sequence learning: perceptual rather than conceptual? Psychological Research, 71, $140-151$.

Deroost, N., \& Soetens, E. (2006). Spatial processing and perceptual sequence learning in SRT tasks. Experimental Psychology, 53, $16-30$.

Hoffmann, J., \& Koch, I. (1998). Implicit learning of loosely defined structures. In M. A. Stadler \& P. A. Frensch (Eds.), Handbook of Implicit Learning (pp. 161-199). Thousand Oaks: Sage Publications.

Hoffmann, J., Sebald, A., \& Stoecker, C. (2001). Irrelevant response effects improve serial learning in serial reaction time tasks. Journal of Experimental Psychology: Learning Memory and Cognition, 27, 470-482.

Hsiao, A. T., \& Reber, A. S. (2001). The dual-task SRT procedure: finetuning the timing. Psychonomic Bulletin \& Review, 8, 336-342.

Jiménez, L., \& Mendez, C. (1999). Which attention is needed for implicit sequence learning? Journal of Experimental Psychology: Learning Memory and Cognition, 25, 236-259.

Keele, S. W., Ivry, R., Mayr, U., Hazeltine, E., \& Heuer, H. (2003). The cognitive and neural architecture of sequence representation. Psychological Review, 110, 316-339.

Koch, I., \& Hoffmann, J. (2000). The role of stimulus-based and response-based spatial information in sequence learning. Journal of Experimental Psychology: Learning Memory and Cognition, 26, $863-882$.

Mayr, U. (1996). Spatial attention and implicit sequence learning: evidence for independent learning of spatial and nonspatial sequences. Journal of Experimental Psychology: Learning Memory and Cognition, 22, 350-364.

Meier, B., \& Cock, J. (2010). Are correlated streams of information necessary for implicit sequence learning? Acta Psychologica, 133, 17-27.

Meier, B., \& Cock, J. (2012). The role of cues and stimulus valency in implicit task sequence learning: a task sequence is not enough. In A. L. Magnusson \& D. J. Lindberg (Eds.). Psychology of Performance and Defeat (pp. 155-166). Hauppauge: Nova Science Publisher.

Nemeth, D., Hallgato, E., Janacsek, K., Sandor, T., \& Londe, Z. (2009). Perceptual and motor factors of implicit skill learning. Neuro Report, 20, 1654-1658.

Nissen, M. J., \& Bullemer, P. (1987). Attentional requirements of learning: evidence from performance measures. Cognitive Psychology, 19, 1-32.

Rah, S. K.-Y., Reber, A. S., \& Hsiao, A. T. (2000). Anotherwrinkle on the dual-task SRT experiment: it's probably not dual task. Psychonomic Bulletin \& Review, 7, 309-313.

Riedel, B., \& Burton, A. M. (2006). Auditory sequence learning: differential sensitivity to task relevant and task irrelevant sequences. Psychological Research, 70, 337-344.

Robertson, E. M., Tormos, J. M., Maeda, F., \& Pascual-Leone, A. (2001). The role of the dorsolateral prefrontal cortex during sequence learning is specific for spatial information. Cerebral Cortex, 11, 628-635.

Rowland, L. A., \& Shanks, D. R. (2006). Attention modulates the learning of multiple contingencies. Psychonomic Bulletin \& Review, 13, 643-648.

Schmidtke, V., \& Heuer, H. (1997). Task integration as a factor in secondary-task effects on sequence learning. Psychological Research, 60, 53-71.

Schwarb, H., \& Schumacher, E. H. (2010). Implicit sequence learning is represented by stimulus-response rules. Memory and Cognition, 38, 677-688.

Shin, J. C., \& Ivry, R. B. (2002). Concurrent learning of temporal and spatial sequences. Journal of Experimental Psychology: Learning Memory and Cognition, 28, 445-457. 
Stoecker, C., Sebald, A., \& Hoffmann, J. (2003). The influence of response-effect compatibility in a serial reaction time task. Quarterly Journal of Experimental Psychology, 56, 685-703.

Weiermann, B., Cock, J., \& Meier, B. (2010). What matters in implicit task sequence learning: perceptual stimulus features, task-sets, or correlated streams of information? Journal of Experimental Psychology: Learning Memory and Cognition, 36, $1492-1509$.
Weiermann, B., \& Meier, B. (2012). Implicit task sequence learning with auditory stimuli. Journal of Cognitive Psychology, 24, 468-475.

Ziessler, M., \& Nattkemper, D. (2001). Learning of event sequences is based on response-effect learning: further evidence from a serial reaction task. Journal of Experimental Psychology: Learning Memory and Cognition, 27, 595-613. 